\title{
XMM-Newton observations of SN 1987 A
}

\author{
F. Haberl, U. Geppert, B. Aschenbach, and G. Hasinger
}

\author{
Max-Planck-Institut für extraterrestrische Physik, Giessenbachstraße, 85748 Garching, Germany \\ e-mail: fwh@mpe.mpg.de
}

Received 7 August 2006 / Accepted 13 September 2006

\begin{abstract}
Context. We report on XMM-Newton observations of SN 1987 A in the Large Magellanic Cloud.

Aims. The large collecting area telescopes together with the European Photon Imaging Cameras (EPIC) provide X-ray spectra with unprecedented statistical quality and make it possible to investigate the spectral evolution during the brightening observed since the discovery in X-rays. High resolution spectra from the Reflection Grating Spectrometers yield a complementary view and allow us to perform more detailed investigations of prominent emission lines.

Methods. The X-ray spectra were modeled with two-temperature emission components from a hot plasma in collisional ionization equilibrium and in non-equilibrium (NEI).

Results. We find a temperature for the equilibrium component of $0.24 \pm 0.02 \mathrm{keV}$ in January 2000 and April 2001 which increased to $0.30 \pm 0.02 \mathrm{keV}$ in May 2003 and also an indication for a temperature increase in the hot NEI component from $\sim 2 \mathrm{keV}$ to $\sim 3 \mathrm{keV}$. Emission line ratios inferred from the RGS spectra suggest temperatures as low as $100 \mathrm{eV}$ and an increase in the ionization state of oxygen and neon consistent with the observed temperature increases. The fast readout of the EPIC-pn instrument yields X-ray fluxes free of CCD pile-up effects which we used to normalize pile-up corrections for the published Chandra fluxes. The corrected X-ray light curve of SN $1987 \mathrm{~A}$ in the $0.5-2.0 \mathrm{keV}$ energy band is best represented by a linear increase up to about day 4000 after the explosion and an exponential rise afterwards until the last published Chandra observation on day 6716. Modeling the light curve by emission from the inner ring which is approximated by a circular torus a central density $n_{\mathrm{H}}=1.15 \times 10^{4} \mathrm{~cm}^{-3}$ is found. In this model the forward shock has just passed the center of the torus.

Conclusions. SN 1987 A continues to brighten exponentially in soft X-rays. The X-ray spectra can be represented by pure thermal emission without significant contribution from a compact object yet.
\end{abstract}

Key words. ISM: supernova remnants - stars: supernovae: general - stars: supernovae: individual: SN 1987 A - X-rays: general $\mathrm{X}$-rays: stars

\section{Introduction}

The first supernova observed in 1987 (SN 1987 A) went off in the Large Magellanic Cloud (LMC) on 23 February. This event with observations involving the entire electromagnetic spectrum and the first detection of cosmic neutrinos brought about a number of astrophysical surprises, some of which are puzzling still today. In this paper we concentrate on the observations of soft $(0.1-3 \mathrm{keV}) \mathrm{X}$-ray emission since the explosion, thought to arise from the interaction of shocks with the ambient circumstellar medium, and possibly a central compact object like a neutron star. We present and summarize for the first time the full set of previous data, which to some extent have been re-calibrated, and we add the XMM-Newton observations.

The first attempts to search for soft X-rays were made in August 1987 with sounding rockets carrying imaging telescopes. An upper limit of $1.5 \times 10^{36} \mathrm{erg} \mathrm{s}^{-1}$ could be established which excluded an ambient dense and slow wind typical for a red supergiant wind, but which is still compatible with a blue supergiant (Aschenbach et al. 1987). X-rays from SN 1987 A became clearly visible in the ROSAT images not much before spring 1992 (Beuermann et al. 1994; Gorenstein et al. 1994). The monitoring of SN 1987 A with ROSAT revealed a slow, but steady increase in flux over the first years (Hasinger et al. 1996). The brightening continues since then but with increasing pace. (Burrows et al. 2000; Park et al. 2002, 2004, 2005). Chandra has taken images of SN 1987 A since late 1999, which show a ring-like emission region steadily growing in diameter. Details of the ring structure became apparent in the meantime. These structural features are correlated with the optical hot spots, first discovered in earlier images taken with the Hubble Space Telescope (HST). These optically bright spots evolve quite rapidly and they now seem to completely engulf the so-called inner ring (Lawrence et al. 2000; McCray 2005).

The ring with its fairly high matter density existed before the explosion and was detected by re-emitted light of the prompt supernova EUV flash. A substantial heating, up to X-ray temperatures, of the ring was predicted for the period between 2002 and 2010 when the supernova shock wave would have reached and entered the ring. Both the optical and X-ray images and the $\mathrm{X}$-ray light curve demonstrate that this state has been arrived at. Hope is that we can learn more about the details of the interaction process, the origin of the ring as well as of the progenitor star and its evolution by X-ray spectroscopy.

The medium energy resolution X-ray spectrum measured by the Chandra ACIS detector is composed of two emission components with temperatures $k T$ of $\sim 2.4 \mathrm{keV}$ and $\sim 0.22 \mathrm{keV}$, which sometimes are interpreted as contributions from the blast wave shock and the decelerated slow shock, respectively (Michael et al. 2002; Park et al. 2004). The strong brightness increase made it also possible to obtain high resolution X-ray spectra with the Chandra LETG. A total exposure of $289 \mathrm{ks}$ was accumulated between August 26 and September 5, 2004. Inferred temperatures from the emission line ratios range from 0.1 to $2 \mathrm{keV}$ and 
Table 1. XMM-Newton observations of SN 1987 A and data selection.

\begin{tabular}{lcccccrr}
\hline \hline Instrument & $\begin{array}{c}\text { Read-out } \\
\text { Mode }\end{array}$ & Filter & Sat. Revol. & Date & Time (UT) & $\begin{array}{c}\text { Net Exp } \\
{[\mathrm{s}]}\end{array}$ & Counts $^{2}$ \\
\hline EPIC-pn & FF, 73 ms & Medium & 21 & 2000 Jan. 19/20 & $16: 19-04: 28$ & 23250 & 2194 \\
EPIC-pn & FF, 73 ms & Medium & 22 & 2000 Jan. 21 & $15: 38-19: 34$ & 5789 & 518 \\
EPIC-pn & FF, 73 ms & Thin & 22 & 2000 Jan. 21/22 & $20: 32-12: 02$ & 30990 & 3060 \\
\hline EPIC-pn & FF, 73 ms & Medium & 244 & 2001 Apr. 8/9 & $20: 35-07: 14$ & 24534 & 3952 \\
\hline EPIC-pn & SW, 6 ms & Medium & 626 & 2003 May 10/11 & $16: 41-23: 37$ & 55855 & 25609 \\
EPIC-MOS1 & FF, 2.6 s & Medium & & & $16: 35-23: 37$ & 101520 & 16020 \\
EPIC-MOS2 & FF, 2.6 s & Medium & & & $16: 35-23: 37$ & 101510 & 16018 \\
RGS1 & Spectro & - & & & $11: 42-23: 38$ & 112160 & 2509 \\
RGS2 & Spectro & - & & & $10: 47-23: 38$ & 111670 & 3358 \\
\hline
\end{tabular}

${ }^{1}$ Net exposures for the observations from revolutions 21/22 were derived from a comparison of the spectrum of N 157 B with that obtained from the ROSAT PSPC due to missing timing information in the early data (Haberl et al. 2001). ${ }^{2}$ Net counts as used for the spectral analysis in the following energy bands: $0.2-9.0 \mathrm{keV}$ for the EPIC instruments (except for EPIC-pn during revolutions 21/22 when we adopted 0.3-9.0 keV due to the higher low-energy threshold used at the beginning of the performance verification phase); $0.3-2.0 \mathrm{keV}$ for RGS.

expansion velocities are found between 300 and $1700 \mathrm{~km} \mathrm{~s}^{-1}$ (Zhekov et al. 2005).

One other outstanding issue is the existence and the time of appearance of a central compact remnant like a neutron star. So far the results have been negative. The most stringent upper limits for an X-ray point source in the newly born supernova remnant are based on the analysis of Chandra images in the $2-10 \mathrm{keV}$ band with $L_{\mathrm{x}}<1.5 \times 10^{34} \mathrm{erg} \mathrm{s}^{-1}$ (Park et al. 2004) and on INTEGRAL measurements in the $20-60 \mathrm{keV}$ band $\left(L_{\mathrm{x}}<1.1 \times 10^{36} \mathrm{erg} \mathrm{s}^{-1}\right.$, Shtykovskiy et al. 2005). The HST derived optical limit is $L_{\mathrm{opt}}<5 \times 10^{33} \mathrm{erg} \mathrm{s}^{-1}$ (Graves et al. 2005).

XMM-Newton observed SN 1987 A on several occasions. Here, we report the results of the spectral analysis of the data with the highest statistical quality available to date, making use of the EPIC cameras and the RGS spectrometers.

\section{X-ray observations}

XMM-Newton (Jansen et al. 2001) observations from three epochs were used to investigate the spectral evolution of SN 1987 A (for observation details see Table 1). The 30 Doradus region in the Large Magellanic Cloud was selected for firstlight including SN $1987 \mathrm{~A}$ at an off-axis angle of about $7 \mathrm{arcmin}$ (Dennerl et al. 2001). Five exposures were taken with the EPIC-pn instrument (Strüder et al. 2001) in full-frame (FF) imaging mode which we use for spectral analysis as described by Haberl et al. (2001). Exposures with the same instrumental setup (pointing direction, optical blocking filter) were merged resulting in three EPIC-pn "first-light" spectra of SN 1987 A from January 2000. An observation at a second epoch in April 2001 was performed utilizing all instruments on board of XMM-Newton; the CCD cameras EPIC-pn and EPIC-MOS (Turner et al. 2001) and the Reflection Grating Spectrometers (RGS, den Herder et al. 2001). For spectral analysis we use the EPIC-pn data from this observation.

Spectra from all instruments were accumulated from the $\sim 100$ ks observation performed in May 2003 when the source was sufficiently bright such that the long exposure yielded spectra with sufficient statistical quality for all instruments. During this observation EPIC-pn was set to the faster smallwindow (SW) CCD readout mode. For optical light blocking the "medium" filter was selected in the EPIC cameras, except during the last part of the first-light observations, when a thin filter was used.

We screened the data for high background intervals and extracted source and background spectra of SN 1987 A from circular regions with a $25^{\prime \prime}$ radius placed on the source and a nearby point-source free area. For the EPIC-pn spectra singlepixel (PATTERN 0) events were selected while for EPIC-MOS all valid event patterns (PATTERN 0 to 12) were used. The spectra were binned to contain a minimum of 30 and 40 counts per bin for EPIC and RGS, respectively. Table 1 lists net exposures and net counts accumulated for the spectra. The data from satellite revolutions 244 and 626 were processed with SAS release 6.1.0 together with the latest calibration files relevant for spectral analysis of EPIC-pn data (EPN_REDIST_0010.CCF for the re-distribution matrices and XRT3_XAREA_0010.CCF with an update to the mirror effective area). The spectra from revolutions 21/22 were directly taken from the work of Dennerl et al. (2001) and Haberl et al. (2001) together with new response files mentioned above. Spectral fitting was performed using XSPEC (Arnaud 1996) version 11.3.2i.

\section{Spectral analysis}

For studying the spectral evolution of SN 1987 A we concentrate on the EPIC-pn data because they provide the spectra with the highest statistical quality, in particular for the early observations when the source was still comparatively faint. We simultaneously fit the five EPIC-pn spectra with different twotemperature models similar to those applied to Chandra ACIS spectra by Park et al. (2004). The best results were found using a two component thermal model (cf. Table 2) involving both the collisional ionization equilibrium models (XSPEC models MEKAL or RAYMOND, Mewe et al. 1985; Raymond \& Smith 1977) and/or the non-equilibrium model (NEI, Borkowski et al. 2001). For the photo-electric foreground absorption we use a fixed Galactic column density of $N_{\mathrm{H}}=6 \times 10^{20} \mathrm{~cm}^{-2}$ with the elemental abundances given by Wilms et al. (2000) and an LMC column density (free in the fit) with the same abundances relative to $\mathrm{H}$ but reduced by a factor of 2 for each element except $\mathrm{He}$ (also relative to the Wilms et al. abundances). The, in comparison with the solar value of Anders \& Grevesse (1989), lower oxygen abundance of Wilms et al. better fits the EPIC spectra around the oxygen $\mathrm{K}$ absorption edge.

We allowed some key parameters of the models to vary with time, i.e. they are free parameters for the individual spectra of different epochs. These are temperature and normalization (given in the following as emission measure). The ionization timescale in the NEI models was found to be the same within the error limits for the various spectra and therefore it was used just as one single free parameter in the combined fit. Similarly, 
Table 2. Fit quality of two-temperature models.

\begin{tabular}{|c|c|c|c|c|c|c|c|c|c|}
\hline \multirow[t]{2}{*}{ Model $^{\star}$} & \multicolumn{3}{|c|}{ EPIC-pn, all } & \multicolumn{3}{|c|}{ MOS, rev. 626} & \multicolumn{3}{|c|}{ RGS, rev. 626} \\
\hline & $\chi^{2}$ & d.o.f. & $\chi_{r}^{2}$ & $\chi^{2}$ & d.o.f. & $\chi_{r}^{2}$ & $\chi^{2}$ & d.o.f. & $\chi_{r}^{2}$ \\
\hline A: PHABS * VPHABS * (VNEI + VRAYMOND) & 806.5 & 716 & 1.126 & 864.1 & 357 & 2.434 & 799.0 & 580 & 1.377 \\
\hline B: PHABS * VPHABS * (VNEI + VMEKAL) & 804.8 & 716 & 1.124 & 903.6 & 357 & 2.545 & 811.4 & 580 & 1.399 \\
\hline C: $\mathrm{PHABS} *$ VPHABS * $(\mathrm{VNEI}+\mathrm{VNEI})$ & 802.2 & 715 & 1.122 & 890.4 & 357 & 2.508 & 870.2 & 580 & 1.500 \\
\hline D: PHABS * VPHABS * (VMEKAL + VMEKAL) & 858.1 & 717 & 1.197 & 878.4 & 355 & 2.474 & 810.3 & 580 & 1.397 \\
\hline
\end{tabular}

* Model components as available in XSPEC: PHABS represents the Galactic foreground absorption, VPHABS the absorption in the LMC with reduced metal abundances, VRAYMOND and VMEKAL emission from hot plasma in collisional ionization equilibrium with variable abundances and VNEI emission from a non-equilibrium plasma (see also Sect. 3).

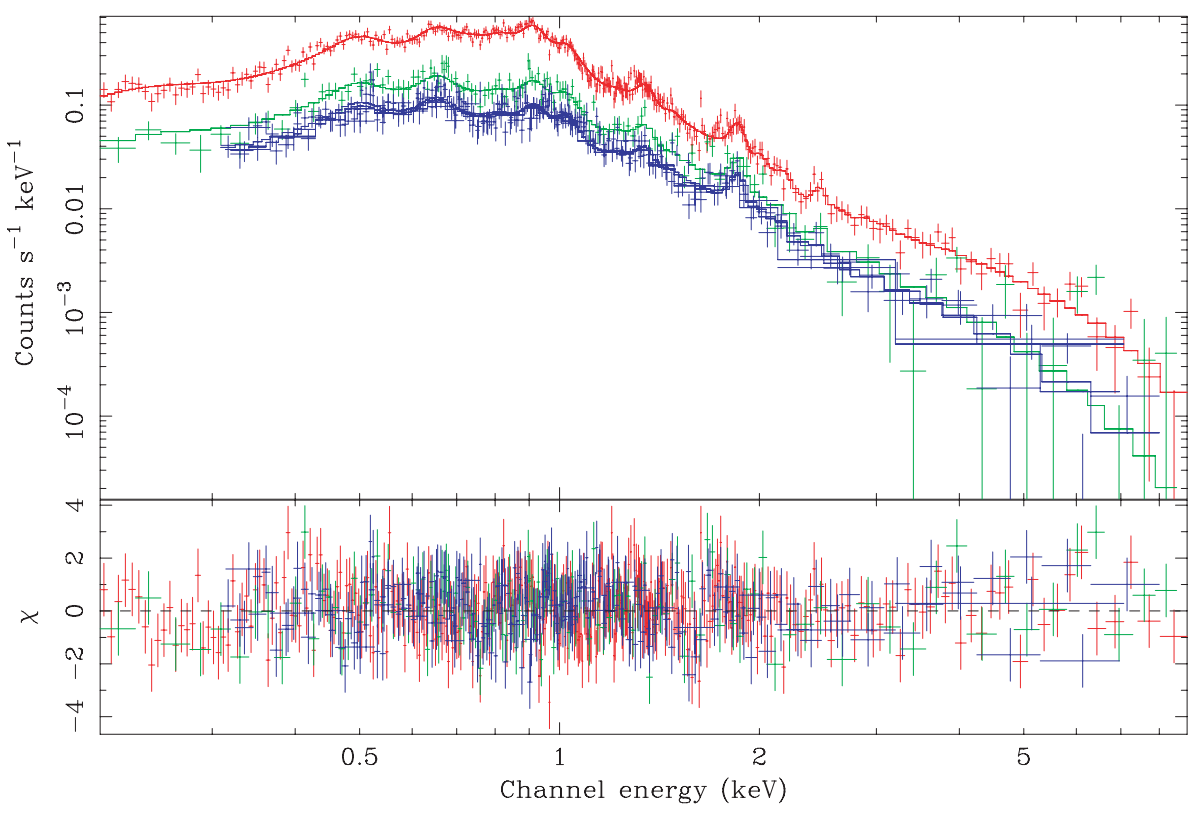

Fig. 1. EPIC-pn spectra of SN 1987 A from Jan. 2000, April 2001 and May 2003 (in increasing intensity). The histograms show the best fit model A as defined in Table 2 and parameters listed in Tables 3 and 4. we assumed that the set of elemental abundances used in the emission models did not change with time as a whole, but we still fit abundances individually for each element. Also for the emission models abundances relative to those given by Wilms et al. (2000) are used. For the three first-light spectra only a constant factor was allowed to vary to account for the uncertainties in the net exposure time. However the spectra agree within $\pm 3 \%$.

The energy resolution of the EPIC instruments does not allow to differentiate between the different two-temperature models. Table 2 summarizes the quality of the fits for the best four models. The combined EPIC-pn fits yield a somewhat smaller reduced $\chi^{2}$ values for models $\mathrm{A}, \mathrm{B}$ and $\mathrm{C}$ which each involve a non-equilibrium model for the high energy part of the spectrum. The EPIC-pn spectrum together with the best fit model A is shown in Fig. 1. We cross-checked the results from the EPIC-pn fits with the EPIC-MOS and RGS spectra from revolution 626 (earlier spectra have insufficient statistics) by folding the model through the corresponding detector response (SAS 6.1.0 version) but optimizing the normalization through a constant factor. The MOS spectra (Fig. 2) are fully compatible with the pn results above $\sim 0.8 \mathrm{keV}$. Below that energy the MOS fits suffer from the changing event redistribution which is not included in the calibration files of SAS 6.1.0. The RGS spectra with their higher energy resolution (Fig. 3) formally exclude model C (this model produces a strong line at $0.512 \mathrm{keV}$ which does not exist in the data).

In the following we present more detailed results for models A and B. The best fit parameters are summarized in Tables 3 and 4. Model A is a combination of NEI and RAYMOND emission components while model B uses NEI and MEKAL. The medium resolution EPIC-pn spectra can not discriminate between these two models, but we prefer RAYMOND rather than MEKAL because the latter produces a S XIV line at $0.512 \mathrm{keV}$ which is not seen in the data and not present in any other model (see RGS spectra in Fig. 3). Apart from this difference the best fit parameters of model A and model B agree very well within their error limits.

For both models there is clear evidence that both the temperature of the soft (MEKAL/RAYMOND) component as well as the temperature of the hard (NEI) component have increased after the second epoch (rev. 244) observation. This can also be directly seen in the shape of the EPIC-pn spectra below $1 \mathrm{keV}$ where line intensity ratios differ between the spectra from revolutions 244 and 626 (Fig. 1). In particular the Ne IX triplet increased in strength with respect to O VIII. The increase in temperature may be related to the progressing equilibration of electron and ion temperatures.

The EPIC-pn derived best fit model A reproduces most of the emission lines resolved in the high resolution RGS spectra, except the O VII He-like triplet near 574 eV (Fig. 3), which suggests that some plasma with temperatures lower than $0.3 \mathrm{keV}$ contributes to the emission. Oxygen line ratios derived from the September 2004 Chandra LETG spectrum, taken about 1.5 years after the last XMM-Newton observation, are consistent with lower temperatures of about $100 \mathrm{eV}$ (Zhekov et al. 2005). For a quantitative comparison of the line strengths of the most prominent emission lines seen in the LETG and RGS spectra we modeled the RGS spectra in a way similar to the work of 

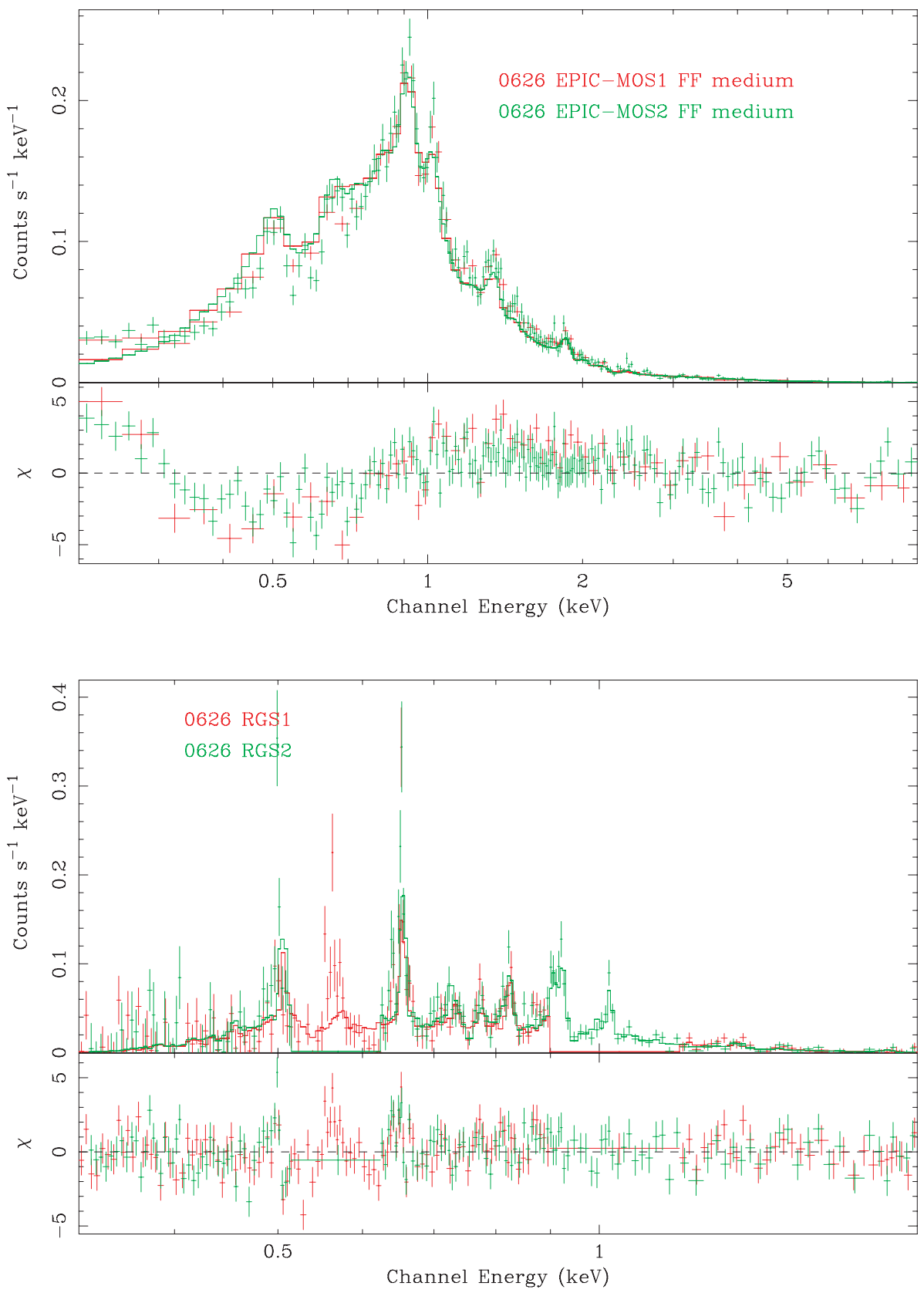

Fig. 2. EPIC-MOS spectra of SN 1987 A from satellite revolution 626 . The histograms show the prediction of model A with the best-fit parameter values of the EPIC-pn spectrum except the normalization (a constant scaling factor of 0.98 and 0.99 for MOS1 and MOS2, respectively).
Fig. 3. RGS spectra of SN 1987 A from satellite revolution 626. The model is as for Fig. 2 with scaling factors 1.01 and 0.98 for RGS1 and RGS2, respectively.
Zhekov et al. (2005) but with the exception that we modeled both RGS spectra simultaneously with an absorbed bremsstrahlung component for the continuum and a set of emission lines with Gaussian line profiles. For completeness, we included all those emission lines which are detected in the RGS spectra of the bright Magellanic Cloud supernova remnants 1E 0102.2-7219 (Rasmussen et al. 2001) and N132D (Behar et al. 2001). This makes sure that no observed emission line is missed in the model as is the case for the plasma models used above. The width of all lines was forced to be the same, i.e. one free parameter. This resulted in a best fit value for the full width at half maximum of $5.3 \pm 1.0 \mathrm{eV}$ which corresponds to a Doppler velocity of $2430 \pm 460 \mathrm{~km} \mathrm{~s}^{-1}$.

All line energies were fixed at the laboratory values, but red-shifted by the LMC Doppler shift of $286 \mathrm{~km} \mathrm{~s}^{-1}$, which was found to be consistent with the LETG spectrum (Zhekov et al. 2005). Leaving the common red-shift for all lines as free parameter in the fits a Doppler velocity of $460 \pm 126 \mathrm{~km} \mathrm{~s}^{-1}$ ( $90 \%$ confidence) is inferred from the RGS spectra. Taking into account the RGS wavelength scale calibration uncertainties of $\sim 200 \mathrm{~km} \mathrm{~s}^{-1}$ the LETG and RGS derived LMC Doppler shifts are consistent with each other. The line fluxes derived for the fixed Doppler velocity of $286 \mathrm{~km} \mathrm{~s}^{-1}$ are listed in Table 5 . We assumed $286 \mathrm{~km} \mathrm{~s}^{-1}$ to allow a direct comparison with the LETG values published by Zhekov et al. (2005). Simultaneously we verified that the line fluxes change only marginally when the best-fit Doppler shift of $460 \mathrm{~km} \mathrm{~s}^{-1}$ is used (the fluxes change by less then $5 \%$ which is less than the statistical errors). As can be seen from the LETG/RGS flux ratios presented in Table 5, all line intensities except the O VII triplet increased over the 1.5 years between the XMM-Newton (May 2003) and Chandra (Sep. 2004) observations, consistent with the trend seen in the EPIC-pn spectra. Although the errors are relatively large there is an indication that the ionization degree of $\mathrm{O}$ and 
Table 3. Results from the two-temperature model fits to the EPIC-pn spectra - the parameters allowed to vary with time. Fluxes are given in units of $10^{-13} \mathrm{erg} \mathrm{cm}^{-2} \mathrm{~s}^{-1}$ and emission measures EM in $10^{58} \mathrm{~cm}^{-3}$.

\begin{tabular}{lccc}
\hline \hline Parameter & $\begin{array}{c}\text { Rev. 626 } \\
\text { May 2003 }\end{array}$ & $\begin{array}{c}\text { Rev. 244 } \\
\text { Apr. 2001 }\end{array}$ & $\begin{array}{c}\text { Rev. 21/22 } \\
\text { Jan. 2000 }\end{array}$ \\
\hline Model A & & & \\
NEI $k T(\mathrm{keV})$ & $3.22_{-0.49}^{+0.53}$ & $1.76_{-0.34}^{+0.68}$ & $2.10_{-0.29}^{+0.35}$ \\
NEI EM & $1.00_{-0.15}^{+0.08}$ & $0.69_{-0.15}^{+0.18}$ & $0.43_{-0.06}^{+0.06}$ \\
RAYMOND $k T(\mathrm{keV)})$ & $0.30_{-0.02}^{+0.02}$ & $0.24_{-0.02}^{+0.02}$ & $0.24_{-0.02}^{+0.02}$ \\
RAYMOND EM & $16.0_{-3.7}^{+3.0}$ & $5.1_{-1.4}^{+1.1}$ & $3.2_{-0.9}^{+0.7}$ \\
Flux (0.5-2.0 keV) & 8.10 & 2.77 & 1.75 \\
Flux (3.0-10.0 keV) & 1.77 & 0.43 & 0.39 \\
Flux (0.2-10.0 keV) & 11.4 & 3.82 & 2.59 \\
\hline Model B & & & \\
NEI $k T(\mathrm{keV})$ & $3.11_{-0.45}^{+0.63}$ & $1.65_{-0.18}^{+0.42}$ & $2.01_{-0.28}^{+0.17}$ \\
NEI EM & $1.03_{-0.14}^{+0.14}$ & $0.74_{-0.15}^{+0.25}$ & $0.45_{-0.05}^{+0.07}$ \\
MEKAL $k T(\mathrm{keV})$ & $0.31_{-0.02}^{+0.02}$ & $0.25_{-0.02}^{+0.03}$ & $0.24_{-0.02}^{+0.01}$ \\
MEKAL EM & $13.4_{-1.3}^{+1.4}$ & $4.0_{-0.9}^{+0.9}$ & $2.6_{-0.6}^{+0.6}$ \\
Flux (0.5-2.0 keV) & 8.11 & 2.78 & 1.75 \\
Flux (3.0-10.0 keV) & 1.74 & 0.40 & 0.38 \\
Flux (0.2-10.0 keV) & 11.4 & 3.79 & 2.58 \\
\hline
\end{tabular}

Table 4. Results from the two-temperature model fits to the EPIC-pn spectra - the parameters assumed to be constant with time.

\begin{tabular}{lcc}
\hline \hline Parameter & Model & Model \\
& $\mathrm{A}$ & $\mathrm{B}$ \\
\hline Galactic $N_{\mathrm{H}}\left(10^{21} \mathrm{~cm}^{-2}\right.$, fixed) & 0.6 & 0.6 \\
$\mathrm{LMC} N_{\mathrm{H}}\left(10^{21} \mathrm{~cm}^{-2}\right)$ & $2.50_{-0.33}^{+0.19}$ & $2.27_{-0.22}^{+0.19}$ \\
$n_{\mathrm{e}} t\left(10^{10} \mathrm{~s} \mathrm{~cm}^{-3}\right)$ & $5.7_{-1.2}^{+1.4}$ & $5.8_{-1.3}^{+1.8}$ \\
$\mathrm{He}($ fixed) & 2.57 & 2.57 \\
$\mathrm{C}$ (fixed) & 0.09 & 0.09 \\
$\mathrm{~N}$ & $0.80_{-0.27}^{+0.52}$ & $0.95_{-0.38}^{+0.57}$ \\
$\mathrm{O}$ & $0.066_{-0.013}^{+0.030}$ & $0.099_{-0.020}^{+0.017}$ \\
$\mathrm{Ne}$ & $0.24_{-0.04}^{+0.07}$ & $0.18_{-0.02}^{+0.04}$ \\
$\mathrm{Mg}$ & $0.27_{-0.06}^{+0.08}$ & $0.24_{-0.07}^{+0.04}$ \\
$\mathrm{Si}$ & $0.66_{-0.12}^{+0.16}$ & $0.61_{-0.12}^{+0.07}$ \\
$\mathrm{~S}$ & $0.32_{-0.18}^{+0.20}$ & $0.31_{-0.16}^{+0.17}$ \\
$\mathrm{Ar}$ (fixed) & 0.54 & 0.54 \\
$\mathrm{Ca}$ (fixed) & 0.34 & 0.34 \\
$\mathrm{Fe}$ & $0.042_{-0.009}^{+0.013}$ & $0.058_{-0.013}^{+0.008}$ \\
$\mathrm{Ni}$ (fixed) & 0.62 & 0.62 \\
\hline
\end{tabular}

Ne has increased from the XMM-Newton to the later Chandra observations.

As shown in Table 2 the fit with two ionization equilibrium models, i.e. model $\mathrm{D}$, cannot really be excluded based on the values of $\chi_{r}^{2}$, but the models $\mathrm{A}$ and $\mathrm{B}$ involving non-equilibrium models appear to fit the data slightly better. In either case the low temperature component is in equilibrium, which means that the ionization parameter $\log \left(n_{\mathrm{e}} \cdot t\right)>12$, whereas it is much lower by about a factor of 18 for the high temperature component $\left(\log \left(n_{\mathrm{e}} \cdot t\right)=10.76\right)$. This could mean that the high temperature component has formed predominantly in a region with a density lower by that factor. We note that Zhekov et al. (2005) from the September 2004 LETG observation, which took place
Table 5. RGS line fluxes for prominent emission lines.

\begin{tabular}{|c|c|c|c|}
\hline Line & $\begin{array}{c}\text { Energy } \\
{[\mathrm{eV}]}\end{array}$ & $\begin{array}{c}\text { Flux }^{\star} \\
{\left[\text { photons } \mathrm{cm}^{-2} \mathrm{~s}^{-1}\right]}\end{array}$ & $\begin{array}{c}\text { Ratio } \\
\text { LETG/RGS }\end{array}$ \\
\hline N VII & 500 & $29.0_{-35}^{+3.1} \times 10^{-6}$ & $1.53 \pm 0.26$ \\
\hline O VII & $574 / 569 / 561$ & $42.9_{-7.1}^{+7.0} \times 10^{-6}$ & $0.81 \pm 0.19$ \\
\hline O VIII & 654 & $43.5_{-3.4}^{+2.8} \times 10^{-6}$ & $1.17 \pm 0.13$ \\
\hline O VII & 666 & $6.2_{-2.2}^{+2.2} \times 10^{-6}$ & - \\
\hline O VIII & 775 & $8.9_{-1.6}^{+2.5} \times 10^{-6}$ & $2.26 \pm 0.69$ \\
\hline $\mathrm{Ne} I X$ & $922 / 915 / 905$ & $45.4_{-5.4}^{-5.0} \times 10^{-6}$ & $1.36 \pm 0.19$ \\
\hline $\mathrm{NeX}$ & 1022 & $19.6_{-31}^{+3.4} \times 10^{-6}$ & $1.86 \pm 0.32$ \\
\hline
\end{tabular}

^ Line fluxes with $1 \sigma$ errors. For the He-like ion triplets the sum of the three lines is given.

about 1.5 years later than the XMM-Newton observation discussed here, find the low temperature component in equilibrium and the high temperature component out of ionization equilibrium with an ionization parameter $\log \left(n_{\mathrm{e}} \cdot t\right)=11.23$, which is a factor of about three higher than the XMM-Newton value. Apart from the separation in observation time the difference between Chandra and XMM-Newton here is that the Chandra analysis is restricted to evaluation of the emission lines and that Zhekov et al. (2005) have used a different emission model, which makes use of a continuous distribution of shock velocities with the ionization parameter rigidly coupled to the post shock temperature. In the following we discuss a possible consequence if the high temperature component would be significantly out of ionization equilibrium.

The region in and close to the equatorial plane of the inner ring is likely to have a higher density than the regions well above and below, which suggests that the low-temperature component is dominated by emission from the equatorial plane, whereas the high-temperature component is preferentially created in regions way off the plane. This would be an alternative to the association of the two temperatures with the forward and reverse shocks. A very simplified check can be made by comparing the emission measures of the two components, which show a ratio of $16 / 1$ for model A. If assumed to be disk-like the fractional emission volume of the low-temperature component scales with the ratio of the height of the inner ring and the radius of the inner ring. We take the ring height to be the same as the ring width which can be determined from the early optical/UV image as about $1 / 15$ of the radius (Panagia \& Gilmozzi 1991). Taking the density ratio as above, i.e. $18 / 1$ and the volume ratio as $1 / 15$ the emission measure ratio is expected to be $18 \cdot 18 / 15=22 / 1$ in comparison with the measured value of 16/1. Given the uncertainties in these numbers, in particular the ring width, and the spatial structure, which is certainly present in the matter distribution, the similarity between measurement and expectation is encouraging.

For determining the elemental abundances we followed the procedure applied to the Chandra LETG data (Zhekov et al. 2005). Because of the fairly low sensitivity of the X-ray instruments values of some abundances were fixed, i.e. for $\mathrm{He}, \mathrm{C}, \mathrm{Ar}$, $\mathrm{Ca}$, and $\mathrm{Ni}$. The abundances of the remaining seven elements $\mathrm{N}$, $\mathrm{O}, \mathrm{Ne}, \mathrm{Mg}, \mathrm{Si}, \mathrm{S}$ and $\mathrm{Fe}$ are free parameters for the fits. Our results on the relative abundances and the results which Zhekov et al. (2005) derived from the Chandra LETG measurements agree very well, despite the fact that the observations were taken at times differing by about 1.5 years. There are, however, two exceptions. We find that the abundance of $\mathrm{Si}$ is about 2 to 3 times 
higher in the earlier XMM-Newton observations. Either this is related to the models or the $\mathrm{Si}$ abundance in the region of the inner ring close to its core is lower than at its inner edge. Besides that the XMM-Newton Si abundance (0.54-0.82) is also larger than what is generally being quoted as the general $\mathrm{Si}$ abundance in the LMC (0.31). The second mismatch occurs for Fe, which is found to be significantly under-abundant in both measurements, but the LETG derived Fe abundance is at least a factor of about two higher than the XMM-Newton result. Does the Fe abundance increase towards the core of the inner ring?

One of the remarkable features of the inner ring is the overabundance of $\mathrm{N}(1.63)$ and the under-abundance of $\mathrm{O}(0.18)$ with a ratio of about 9 . This ratio is consistent with the XMM-Newton measurements with the abundances of $\mathrm{N} \sim 0.8$ and of $\mathrm{O} \sim 0.066$ yielding a ratio of about $12.1 \pm 6$ (cf. Table 4$)$, although the individual values of the $\mathrm{N}$ and $\mathrm{O}$ abundances are both lower by about a factor of 2 to 2.5. In general, the abundances of all elements except that of $\mathrm{He}$ are lower than solar. But it seems that the seven elements which were set free in the fits fall into three groups, which are $\mathrm{N}$ and $\mathrm{Si}$ in group one with an average abundance value of $0.73, \mathrm{Ne}, \mathrm{Mg}$ and $\mathrm{S}$ in group 2 with an average abundance of 0.28 , and $\mathrm{O}$ and $\mathrm{Fe}$ in group 3 with the very low abundance of 0.054 . Given the error boundaries there is no overlap between these three groups. If this distinction is not a chance coincidence it might tell us something about the creation of the inner ring and/or the progenitor. Within each group, though, the abundance ratio conforms to solar values, i.e. the ratio of $\mathrm{O} / \mathrm{Fe}$ is solar-like, as is that of N/Si, as well as the ratio for the elements in group 3. It is unlikely that these abundances have resulted from a depletion process, which leaves the creation process of the elements in the progenitor star as the most likely origin.

At last we discuss the column density, the LMC $N_{\mathrm{H}}$ of $\left(2.50_{-0.33}^{+0.19}\right) \times 10^{21} \mathrm{~cm}^{-2}$ for the photo-electric absorption just in the LMC. This can be compared with the total column density throughout the LMC, which is measured in $\mathrm{HI}$ to be $2.53 \times 10^{21} \mathrm{~cm}^{-2}$ (Brüns et al. 2005). The agreement means that either SN $1987 \mathrm{~A}$ is located at the far side of the LMC or that it shows intrinsic absorption by matter in front and close to it. Actually the X-ray measurement can be used to set an upper limit for cold X-ray absorbing matter at distances from the explosion site greater than the radius of the optical ring, which is $\sim 7 \times 10^{17} \mathrm{~cm}$. If the base of an earlier wind of the progenitor star with a $^{-2}$ profile would be located just outside the inner ring the upper limit of the base density is $4270 \mathrm{~cm}^{-3}$. For a stellar wind with a velocity of $10 \mathrm{~km} \mathrm{~s}^{-1}$ this density requires a fairly large stellar mass loss rate of $5.6 \times 10^{-4}$ solar masses per year. This value is larger by a factor of about six than the upper end of the mass loss range derived from radio observations of type II supernovae (Weiler et al. 2005).

In that sense it cannot be excluded that the entire absorption is due to circumstellar absorption either from a single star progenitor or a pre-explosion binary system. It is curious, though, that this amount of absorption is so close to the LMC total column density. But even if the circumstellar absorption would be just a tenth of the total, which would be compatible with mass loss rates known for red supergiants, the base of the wind can stand close to the inner ring, which would support the suggestion that the ring is a product of the interaction of the winds of the red and blue supergiants. Of course structured mass loss in a binary is still not excluded. Whether there is such a high density, spherically symmetric shell just outside the inner ring radius is likely to be decided by observations of the soft X-ray light-curve over the next couple of years, when the shock wave has passed the inner ring.

\section{X-ray light curve}

\subsection{Data}

Since the discovery in 1994 the soft X-ray flux has been monitored first by ROSAT and then by Chandra and XMM-Newton. Hasinger et al. (1996) have summarized the early ROSAT data. For constructing a self-consistent light curve the energy and flux cross-calibration of the various instruments used are needed to be known to a level possibly comparable to the statistical errors. In the following we make an attempt to recalibrate all previous data taken including ROSAT and Chandra. Using the best fit model from the EPIC-pn first-light spectra we re-determined the count rate to flux conversion factor to $9.05 \times 10^{10} \mathrm{PSPC}$ counts/(erg cm $\left.\mathrm{cm}^{-2} \mathrm{~s}^{-1}\right)$ and also add the fluxes from five HRI and one PSPC observations performed up to nearly day 4000 after explosion, which have not been published previously but are in the ROSAT archive.

Chandra performed a regular monitoring of SN 1987 A since the launch of the observatory with published fluxes before the last XMM-Newton observation up to day 5800 (Park et al. 2004). Extrapolating the exponential flux increase detected by Chandra to the epoch of the last XMM-Newton observation we compute a significantly lower flux than observed with XMM-Newton. To investigate if this is caused by a faster brightening of the source or by an instrumental effect we analyzed two more Chandra observations which are available in the public archive and which were performed after the last XMM-Newton observation. We determined count rates in the $0.5-2.0 \mathrm{keV}$ band and apply the same flux conversion factor as Park et al. (2004) did for their last Chandra observation. The two additional flux values follow nicely the extrapolation of the Chandra light curve demonstrating that the different fluxes given by the two instruments are caused by a systematic effect. Park et al. (2004) state in their paper that they did not correct the Chandra ACIS fluxes for CCD pile-up effects which they estimate to be $<10 \%$. Pile-up of photons causes flux losses and is therefore the most likely origin for the lower Chandra fluxes. In contrast the count rate for the EPIC-pn is far from any pile-up limit, especially during the SW mode observation. In addition, pile-up effects should be reduced for the earlier observations when the fluxes were lower, consistent with the better agreement between XMM-Newton and Chandra fluxes derived from those observations.

We therefore suggest that the lower fluxes derived from Chandra ACIS data are due to pile-up and we have corrected the data for the flux losses following the Chandra users handbook for the dependence of the pile-up fraction on the photon rate with a normalization using the last XMM-Newton observation as reference. We find a pile-up correction for day 6158 of about $22 \%$, which can clearly not be neglected. Three more Chandra fluxes were published by Park et al. (2005). During those observations the CCD readout time was reduced by a factor of about two to reduce pile-up effects. However, for the last Chandra observations the intensity increased by more than a factor of two compared to day 6158 and therefore results in similar pile-up losses. We corrected also these last three Chandra fluxes as before, taking also into account the reduced count rate per readout frame.

The final, corrected X-ray light curve in the $0.5-2.0 \mathrm{keV}$ band is presented in Fig. 4 with the fluxes listed in Table 6. The flux changes linearly with time until about day 3500 and continues to increase exponentially until day 6700 . The insert shows the light curve on a lin-log scale together with the prediction constructed from a model of the matter distribution inside the volume run over by the blast wave. 


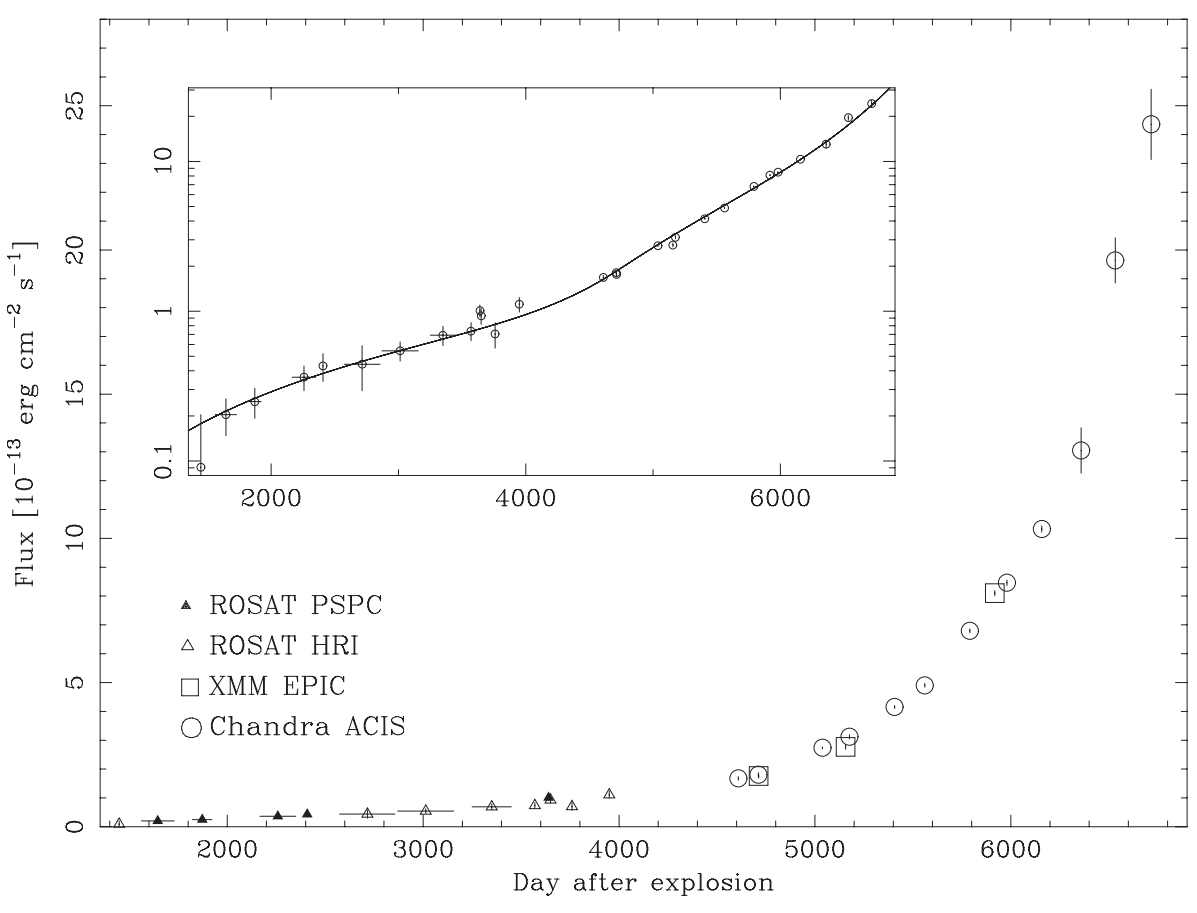

Fig. 4. X-ray light curve of SN $1987 \mathrm{~A}$ in the $0.5-2.0 \mathrm{keV}$ band. Chandra ACIS-S fluxes were corrected for pile-up losses as described in Sect. 4. The insert shows the light curve in logarithmic intensity scale together with the model described in Sect. 4.
Table 6. X-ray fluxes of SN 1987 A.

\begin{tabular}{llcc}
\hline \hline $\begin{array}{l}\text { Age } \\
\text { [days] }\end{array}$ & Instrument & $\begin{array}{c}0.5-2.0 \mathrm{keV} \mathrm{Flux} \\
{\left[10^{-13} \mathrm{erg} \mathrm{cm}^{-2} \mathrm{~s}^{-1}\right]}\end{array}$ & $\begin{array}{c}\text { Pile-up } \\
\text { correction }\end{array}$ \\
\hline 1448 & ROSAT HRI & $0.091 \pm 0.113$ & - \\
1645 & ROSAT PSPC & $0.204 \pm 0.057$ & - \\
1872 & ROSAT PSPC & $0.250 \pm 0.057$ & - \\
2258 & ROSAT PSPC & $0.363 \pm 0.068$ & - \\
2408 & ROSAT PSPC & $0.431 \pm 0.091$ & - \\
2715 & ROSAT HRI & $0.442 \pm 0.147$ & - \\
3013 & ROSAT HRI & $0.544 \pm 0.079$ & - \\
3350 & ROSAT HRI & $0.69 \pm 0.10$ & - \\
3570 & ROSAT HRI & $0.74 \pm 0.10$ & - \\
3640 & ROSAT PSPC & $1.01 \pm 0.09$ & - \\
3650 & ROSAT HRI & $0.93 \pm 0.11$ & - \\
3760 & ROSAT HRI & $0.70 \pm 0.14$ & - \\
3950 & ROSAT HRI & $1.11 \pm 0.12$ & - \\
4609 & Chandra ACIS & $1.68 \pm 0.06$ & 1.035 \\
4711 & Chandra ACIS & $1.81 \pm 0.07$ & 1.038 \\
4712 & XMM EPIC-pn & $1.76 \pm 0.07$ & - \\
5038 & Chandra ACIS & $2.74 \pm 0.03$ & 1.057 \\
5156 & XMM EPIC-pn & $2.77 \pm 0.08$ & - \\
5176 & Chandra ACIS & $3.12 \pm 0.07$ & 1.065 \\
5407 & Chandra ACIS & $4.15 \pm 0.05$ & 1.087 \\
5561 & Chandra ACIS & $4.91 \pm 0.07$ & 1.102 \\
5791 & Chandra ACIS & $6.79 \pm 0.07$ & 1.142 \\
5918 & XMM EPIC-pn & $8.10 \pm 0.09$ & - \\
5980 & Chandra ACIS & $8.46 \pm 0.09$ & 1.177 \\
6158 & Chandra ACIS & $10.32 \pm 0.11$ & 1.216 \\
6359 & Chandra ACIS & $13.05 \pm 0.78$ & 1.136 \\
6533 & Chandra ACIS & $19.64 \pm 0.79$ & 1.205 \\
6716 & Chandra ACIS & $24.35 \pm 1.22$ & 1.255 \\
\hline
\end{tabular}

So far we have concentrated on the soft X-ray supernova light curve but Chandra and XMM-Newton also allow to derive a hard X-ray light curve for the band 3-10 keV. The three XMM-Newton data points on days 4712, 5156 and 5918 agree very well with the Chandra data points given by Park et al. (2005) and confirm the fairly slow increase of the hard flux compared to the soft emission increase up to day 5918.

\subsection{Model}

The emission from SN 1987 A is obviously thermal, so that the observed flux is proportional to the cooling function and the emission measure and inversely proportional to the distance squared. For the distance we take $50 \mathrm{kpc}$. The value of the cooling function is taken from the observational best fits to the spectra; the observed temporal changes in temperature have a very small effect on the cooling function in the energy band of $0.5-2 \mathrm{keV}$, so that we take the cooling function in the band to be constant in time for the observations at hand. Therefore the shape of the SN 1987 A soft X-ray light curve is determined by the emission measure, i.e. the matter density squared integrated over the volume that has been encompassed by the forward shock or blast wave.

The key element is to find a reasonable description for the spatial distribution of the matter density. We assume the inner ring to be a circular torus with a radius of $r_{\mathrm{c}}$ measured to be $6.2 \times 10^{17} \mathrm{~cm}\left(0.83^{\prime \prime}\right)$ (Panagia \& Gilmozzi 1991), a circular cross section and the origin coincident with the explosion site. The density distribution is independent of the azimuth along the torus perimeter - it is circular symmetric. Within the torus the density $n_{\mathrm{H}}$ is described by a Gaussian, i.e. $n_{\mathrm{H}} \propto \exp \left[-\left(\Delta r / h_{\mathrm{i}}\right)^{2}\right]$, with $\Delta \mathrm{r}$ being the shortest distance from the torus center line, not the torus origin, though. At some torus radius $r_{\mathrm{t}}$ a transition from the Gaussian form to a simple exponential dependence occurs, i.e. $n_{\mathrm{H}} \propto \exp \left[-\left(\Delta r / h_{\mathrm{o}}\right)\right]$ for $\Delta r \geq r_{\mathrm{t}}$ (see Fig. 5). The exponential dependence is further modified by a factor of $1 / r$ where $r$ is the distance from the origin. The two forms are normalized to each other such that a smooth transition in density occurs at $\Delta r=r_{\mathrm{t}}$. In addition to $h_{\mathrm{i}}, h_{\mathrm{o}}$ and $r_{\mathrm{t}}$, which are to be determined, the location of the blast wave front $r_{\mathrm{e}}$ at some specified epoch is a free parameter. For this paper we assume the blast wave velocity to be constant in time up to day 7000 . The shocked matter or the X-ray emitting volume elements, are assumed to expand radially and adiabatic with a speed of three quarters of the blast wave velocity.

An acceptable fit to the data (cf. Fig. 4) has been obtained for $r_{\mathrm{e}}=6.3 \times 10^{17} \mathrm{~cm}\left(0.84^{\prime \prime}\right)$ at day 7000 , which means that 


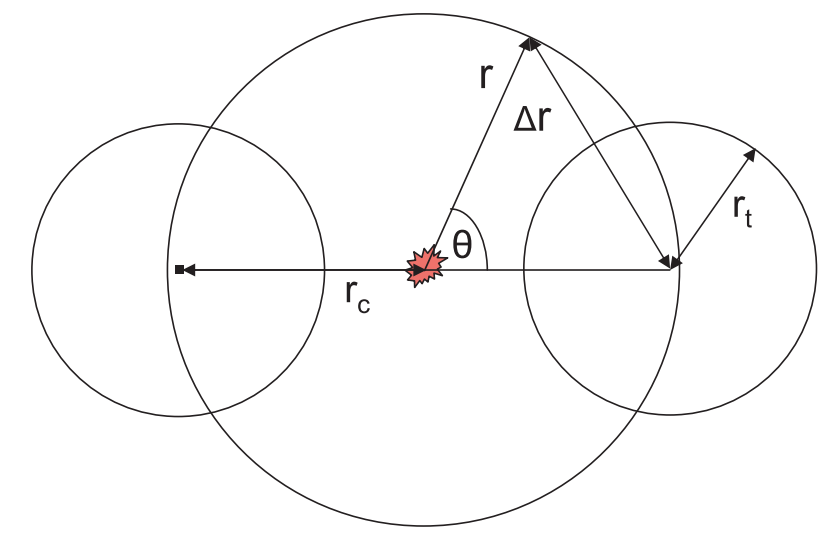

Fig. 5. Sketch used to model the matter distribution around SN 1987 A. The matter is arranged in a circular torus of radius $r_{\mathrm{c}}$; lines of constant density form circles which build up the torus. For torus circles up to radius $r_{\mathrm{t}}$ the distribution follows a Gaussian which has its peak at $r_{\mathrm{c}}$ and falls off with increasing $\Delta r$. Outside of $r_{\mathrm{t}}$ the distribution is roughly exponential which peaks at $r_{\mathrm{t}}$ and decreases with increasing $\Delta r$. For each location in space $(r, \Theta, \phi)$ the density is defined according to this scheme (no dependence on $\phi$ ). The big circle represents the radius of the forward shock wave at day 7000, which has just passed the torus center.

the forward shock has just passed the center of the torus. This also means that the mean blast wave shock velocity over the past 7000 days is $10400 \mathrm{~km} \mathrm{~s}^{-1}$ for a distance of $50 \mathrm{kpc}$. The transition radius $r_{\mathrm{t}}=3.6 \times 10^{17} \mathrm{~cm}\left(0.48^{\prime \prime}\right)$, so that the region inside the torus in which the Gaussian density distribution prevails is roughly half of the torus radius $r_{\mathrm{c}}$. The scale height is $h_{\mathrm{i}}=2.1 \times 10^{17} \mathrm{~cm}\left(0.28^{\prime \prime}\right)$. The scale height of the exponential "atmosphere" of the ring is $h_{\mathrm{o}}=2.6 \times 10^{17} \mathrm{~cm}\left(0.35^{\prime \prime}\right)$. From adjusting the measured and the best-fit fluxes we get a density of $n_{\mathrm{H}, \mathrm{c}}=1.15 \times 10^{4} \mathrm{~cm}^{-3}$ for the innermost core of the ring. This number can be compared with the ring electron density of $n_{\mathrm{e}}=(2-4) \times 10^{4} \mathrm{~cm}^{-3}$ derived from the early optical/UV measurements by Fransson et al. (1989). The ring width, which shows up in these optical/UV measurements, is $6.7 \times 10^{16} \mathrm{~cm}$ $\left(0.09^{\prime \prime}\right)$ (Panagia \& Gilmozzi 1991) and Fransson et al. (1989) estimate for the included mass $0.03-0.05 M_{\odot}$. Our model gives for that limited region a mass of 0.065 solar masses, which is slightly higher. But this number is very sensitive on the exact width of the ring because of the Gaussian density dependence. The total mass overrun by the forward shock wave is, however, much greater and amounts to $0.45 M_{\odot}$, with $90 \%$ of the mass contained in the much wider ring and $10 \%$ in the exponential "atmosphere". The total mass around SN 1987 A out to day 7000 already exceeds the previously quoted mass of the inner ring by a factor of 10 , which may have some relevance to the models explaining the origin of the ring, as far as the energetics are concerned. If the upstream density distribution beyond day 7000 follows the functional forms given above, a total mass of $1.3 M_{\odot}$ will be involved. The soft X-ray light curve is predicted to flatten in mid 2006 and will reach its maximum luminosity of $2 \times 10^{36} \mathrm{erg} / \mathrm{s}$ in early 2009 .

Unacceptable fits to the data, either in the early part or in the late part of the light curve, become apparent for changes of $r_{\mathrm{e}}, r_{\mathrm{t}}$ and $h_{\mathrm{i}}$ exceeding 5 to $7 \%$. This error range is so small basically because $r_{\mathrm{c}}$ is fixed and the distribution is steep. This is different for $h_{\mathrm{o}}$, the scale height of the early data; $h_{\mathrm{o}}$ can be changed up to factor of 2 until deviations become significant. The late time light curve is not affected. Within this range of errors the central density changes by up to $30 \%$.
The numbers given above have been derived for a constant blast wave velocity $v_{\mathrm{b}}$, which is certainly not the case according to the latest Chandra results (Park et al. 2004). This will affect our estimates of $n_{\mathrm{H}, \mathrm{c}}$ and $h_{\mathrm{i}}$, as well as $h_{\mathrm{o}}$, because they scale linearly proportional to $v_{\mathrm{b}}$ and inversely proportional to $v_{\mathrm{b}}$, respectively. The slow down of $v_{\mathrm{b}}$ reaches values of up to a factor of 2 to 3 , in the late stage of the expansion, and accordingly $h_{\mathrm{i}}$ is expected be lower and $n_{\mathrm{H}, \mathrm{c}}$ might reach $(2-4) \times 10^{4} \mathrm{~cm}^{-3}$ on the average. In contrast, the estimates of the mass in the ring is only slightly affected and will stay close to the large values given. In a separate paper we will describe the model in more detail and will present results for a model with $v_{\mathrm{b}}$ slowing down in time.

\section{Limits on a compact object}

\subsection{Observations}

Up to now the search for a compact remnant has not revealed any significant evidence for the existence of a neutron star or black hole, which may be due to various reasons. If a pulsar with a wind nebula were created in the explosion one would expect to see some power-law like X-ray spectrum photo-electrically absorbed by the ambient debris of the progenitor's envelope. Depending on the column density the pulsar and its nebula should appear at some time. To overcome the absorption one should look for the emission in the hard X-ray regime first, and XMM-Newton can supply some data and clues.

To estimate the putative contribution from a compact object to the total flux a power-law component with fixed photon index (2.1) was added to model $\mathrm{A}$ in the combined fit to the EPIC-pn spectra. The intensity was not allowed to vary with time, i.e. it was constrained mainly by the first-light spectra in January 2000. The $\chi^{2}$ improved only to 803.5 which formally does not justify the additional free parameters. For $90 \%$ confidence an upper limit for the flux in the power-law component can be derived. For the $0.2-10.0 \mathrm{keV}(0.5-2.0 \mathrm{keV})$ band this results in an observed, i.e. not corrected for absorption, flux of $1.1 \times 10^{-13} \mathrm{erg} \mathrm{cm}^{-2} \mathrm{~s}^{-1}$ $\left(4.4 \times 10^{-14} \mathrm{erg} \mathrm{cm}^{-2} \mathrm{~s}^{-1}\right)$ which corresponds to an absorption corrected luminosity of $5.7 \times 10^{34} \mathrm{erg} \mathrm{s}^{-1}\left(2.1 \times 10^{34} \mathrm{erg} \mathrm{s}^{-1}\right)$ for a distance of $50 \mathrm{kpc}$. This is slightly less than the absorption uncorrected $2.3 \times 10^{34} \mathrm{erg} \mathrm{s}^{-1}$ upper limit in the Chandra images (0.5-2.0 keV, Burrows et al. 2000). Even if a pulsar was created in the explosion and even if the stellar debris is fairly transparent in the meantime there are other reasons why the pulsar has not emerged in the observations.

\subsection{Discussion}

The detection of neutrinos immediately after the appearance of SN 1987 A is taken as proof that in this supernova a (proto-) neutron star was created. Assuming magnetic flux and angular momentum conservation during the collapse a dipolar surface field of the order of $10^{12} \mathrm{G}$ and a rotational period not much smaller than $0.01 \mathrm{~s}$ are expected. Assuming the "standard" parameter for the moment of inertia $I=10^{45} \mathrm{~g} \mathrm{~cm}^{2}$, we calculate the spindown luminosity $L_{\mathrm{sd}}=4 \pi^{2} I \dot{P} / P^{3} \approx 4 \times 10^{52} \dot{P} \mathrm{erg} \mathrm{s}^{-1}$. Since the X-ray luminosity $L_{\mathrm{x}}=10^{-3} L_{\mathrm{sd}}$ (Becker \& Trümper 1997; Gil et al. 2006), we find a lower limit of $\dot{P} \geq 10^{-15}$ in order for the expected X-ray luminosity of the pulsar to significantly exceed the observed upper limit. If the rotational energy loss of the pulsar is due to magneto-dipole radiation the dipolar surface magnetic field $B_{\mathrm{d}}=2 \times 10^{12}\left(P \dot{P}_{-15}\right)^{1 / 2} \mathrm{G}$, where again the "standard" parameters have been applied, and $\dot{P}_{-15}=\dot{P} / 10^{-15} \mathrm{~s} \mathrm{~s}^{-1}$. 
In order to get the X-ray luminosity of the pulsar larger than $5.7 \times 10^{34} \mathrm{erg} \mathrm{s}^{-1}$, i.e. to enforce a spin-down faster than $\dot{P}_{-15}=1$, the surface dipolar field strength $B_{\mathrm{d}}>2 \times 10^{12} \sqrt{P} \mathrm{G}$, i.e. larger than $2 \times 10^{11} \mathrm{G}$. If the field of the pulsar is lower it would still escape current detection capabilities. Furthermore, in case of SN 1987 A, matter fall-back might have been so powerful that approximately $0.1 M_{\odot}$ have been accreted (Chevalier 1989), which would submerge the initially existing pulsar magnetic fields down to regions close to the crust-core interface of the neutron star. Depending on the equation of state and on the conductive properties of the crust it takes at least 1000 years if not much longer, until the field is re-diffused to the surface with a strength larger than $10^{11} \mathrm{G}$ (see Geppert et al. 1999, and references therein).

The only other possibility we see for the neutron star in SN 1987 A to have a surface magnetic field exceeding $2 \times 10^{11} \mathrm{G}$ is the transformation of thermal into magnetic energy via the strong temperature gradient present in the liquid crust of the young star causing a thermo-magnetic instability (Urpin et al. 1986; Wiebicke \& Geppert 1996). The typical period to reach poloidal surface fields of the order of $10^{12} \mathrm{G}$ however, exceeds certainly $\sim 100 \mathrm{yrs}$. In conclusion, a pulsar powered signature in the spectrum of SN 1987 A is not very likely to appear very soon.

\section{Conclusions}

We present the results from a spectral analysis of XMM-Newton data from SN 1987 A obtained in January 2000, April 2001 and May 2003. The spectra are consistent with thermal emission from the supernova remnant without a significant hard powerlaw component (with an absorption corrected $0.2-10.0 \mathrm{keV}$ luminosity limit of $5.7 \times 10^{34} \mathrm{erg} \mathrm{s}^{-1}$ ) originating from a compact object. Modeling the spectra with two components involving emission from plasma in collisional ionization equilibrium and/or in non-equilibrium yields clear evidence for a temperature rise after the second epoch observation for both components, reaching $\sim 0.3 \mathrm{keV}$ and $\sim 3 \mathrm{keV}$ in the soft and hard component, respectively.

We have collected all available X-ray fluxes in the $0.5-2.0 \mathrm{keV}$ band from ROSAT, XMM-Newton and Chandra for the light curve of SN1987 A up to day 6716 after the explosion. This includes previously unpublished ROSAT data available in the archive and published Chandra fluxes which we corrected for photon pile-up effects present in the CCD spectra. The final light curve shows a linear increase up to about day 4000 and an exponential rise afterwards. We model the light curve with emission from a circular torus around the explosion site which represents the inner ring around SN 1987 A. From the best fit we find that the forward shock just passed the center of the torus and that the mean shock velocity over 7000 days is $10400 \mathrm{~km} \mathrm{~s}^{-1}$. With a core density of the torus of $1.15 \times 10^{4} \mathrm{~cm}^{-3}$ we find a total mass overrun by the forward shock of $0.45 M_{\odot}$. The model, assuming a constant blast wave velocity in its simplest form, predicts a flattening in the X-ray light curve for mid 2006. A new XMM-Newton observation is currently scheduled for the end of 2006 to further monitor the spectral evolution of SN 1987 A.

Acknowledgements. The XMM-Newton project is an ESA Science Mission with instruments and contributions directly funded by ESA Member States and the USA (NASA). The XMM-Newton project is supported by the Bundesministerium für Wirtschaft und Technologie/Deutsches Zentrum für Luft- und Raumfahrt (BMWI/DLR, FKZ 50 OX 0001), the Max-Planck Society and the Heidenhain-Stiftung.

\section{References}

Anders, E., \& Grevesse, N. 1989, Geochim. Cosmochim. Acta, 53, 197 Arnaud, K. A. 1996, in Astronomical Data Analysis Software and Systems V, ASP Conf. Ser., 101, 17

Aschenbach, B., Briel, U. G., Pfeffermann, E., et al. 1987, Nature, 330, 232 Becker, W., \& Trümper, J. 1997, A\&A, 326, 682

Behar, E., Rasmussen, A. P., Griffiths, R. G., et al. 2001, A\&A, 365, L242 Beuermann, K., Brandt, S., \& Pietsch, W. 1994, A\&A, 281, L45

Borkowski, K. J., Lyerly, W. J., \& Reynolds, S. P. 2001, ApJ, 548, 820

Brüns, C., Kerp, J., Staveley-Smith, L., et al. 2005, A\&A, 432, 45

Burrows, D. N., Michael, E., Hwang, U., et al. 2000, ApJ, 543, L149

Chevalier, R. A. 1989, ApJ, 346, 847

den Herder, J. W., Brinkman, A. C., Kahn, S. M., et al. 2001, A\&A, 365, L7

Dennerl, K., Haberl, F., Aschenbach, B., et al. 2001, A\&A, 365, L202

Fransson, C., Cassatella, A., Gilmozzi, R., et al. 1989, ApJ, 336, 429

Geppert, U., Page, D., \& Zannias, T. 1999, A\&A, 345, 847

Gil, J., Melikidze, G. I., \& Zhang, B. 2006, A\&A, 457, L5

Gorenstein, P., Hughes, J. P., \& Tucker, W. H. 1994, ApJ, 420, L25

Graves, G. J. M., Challis, P. M., Chevalier, R. A., et al. 2005, ApJ, 629, 944

Haberl, F., Dennerl, K., Filipović, M. D., et al. 2001, A\&A, 365, L208

Hasinger, G., Aschenbach, B., \& Trümper, J. 1996, A\&A, 312, L9

Jansen, F., Lumb, D., Altieri, B., et al. 2001, A\&A, 365, L1

Lawrence, S. S., Sugerman, B. E., Bouchet, P., et al. 2000, ApJ, 537, L123

McCray, R. 2005, in Cosmic Explosions, On the 10th Anniversary of SN1993J, IAU Colloq., 192, 77

Mewe, R., Gronenschild, E. H. B. M., \& van den Oord, G. H. J. 1985, A\&AS, 62, 197

Michael, E., Zhekov, S., McCray, R., et al. 2002, ApJ, 574, 166

Panagia, N., \& Gilmozzi, R. 1991, in Supernova 1987A and other supernovae, Proceedings of an ESO/EIPC Workshop, Marciana Marina, Isola d'Elba, September 17-22, 1990, Garching: ESO, ed. I. J. Danziger, \& K. Kjaer, 575 Park, S., Burrows, D. N., Garmire, G. P., et al. 2002, ApJ, 567, 314

Park, S., Zhekov, S. A., Burrows, D. N., Garmire, G. P., \& McCray, R. 2004, ApJ, 610, 275

Park, S., Zhekov, S. A., Burrows, D. N., \& McCray, R. 2005, ApJ, 634, L73

Rasmussen, A. P., Behar, E., Kahn, S. M., den Herder, J. W., \& van der Heyden, K. 2001, A\&A, 365, L231

Raymond, J. C., \& Smith, B. W. 1977, ApJS, 35, 419

Shtykovskiy, P. E., Lutovinov, A. A., Gilfanov, M. R., \& Sunyaev, R. A. 2005, Astron. Lett., 31, 258

Strüder, L., Briel, U., Dennerl, K., et al. 2001, A\&A, 365, L18

Turner, M. J. L., Abbey, A., Arnaud, M., et al. 2001, A\&A, 365, L27

Urpin, V. A., Levshakov, S. A., \& Iakovlev, D. G. 1986, MNRAS, 219, 703

Weiler, K. W., Dyk, S. D. V., Sramek, R. A., et al. 2005, in Supernovae as Cosmological Lighthouses, ed. M. Turatto, S. Benetti, L. Zampieri, \& W. Shea, ASP Conf. Ser., 342, 290

Wiebicke, H.-J., \& Geppert, U. 1996, A\&A, 309, 203

Wilms, J., Allen, A., \& McCray, R. 2000, ApJ, 542, 914

Zhekov, S. A., McCray, R., Borkowski, K. J., Burrows, D. N., \& Park, S. 2005, ApJ, 628, L127 